\title{
Thoracic splenosis after thoracoabdominal injury sustained 25 years previously
}

\author{
Levent Cansever'a, Süleyman Ceyhan ${ }^{1}$, Ali Cevat Kutluk' , Yunus Seyrek¹, Firdevs Atabey², Neslihan Akanıl Fener \\ Celalettin İbrahim Kocatürk', Mehmet Ali Bedirhan'1
}

'Department of Thoracic Surgery, Yedikule Chest Disease and Surgery Training and Research Hospital, Istanbul, Turkey ${ }^{2}$ Department of Chest Disease, Yedikule Chest Disease and Surgery Training and Research Hospital, Istanbul, Turkey ${ }^{3}$ Department of Pathology, Yedikule Chest Disease and Surgery Training and Research Hospital, Istanbul, Turkey

\begin{abstract}
Thoracic splenosis may occur after blunt or penetrating trauma of the thoracoabdominal region. It is diagnosed in $65 \%$ of cases of splenic rupture. Trauma-related splenosis is more frequent in the peritoneum, omentum, and mesenterium. The incidence of intrathoracic splenosis is $18 \%$, and the most common location is the pleural space of the left hemithorax. Thoracic splenosis should be considered as a differential diagnosis in patients with multiple lesions in the left hemithorax and a history of splenic trauma involving thoracoabdominal injury.
\end{abstract}

Key Words: surgery, trauma, thoracoscopy/VATS, lung 


\section{Introduction}

Thoracic splenosis was first described by Shaw and Shafi in 1937. The incidence of intrathoracic splenosis is $18 \%$. It is the autotransplantation of splenic tissue secondary to splenectomy or iatrogenic traumatic injury. Thoracic splenosis should be considered as a differential diagnosis in patients with multiple lesions in the left hemithorax and a history of splenic trauma [1-3].

\section{Case Report}

A 58-year-old man was admitted to the hospital for evaluation of chest pain, and nodular lesions were found in the left. Thoracic CT revealed nodular lesions of soft tissue density in the pleural region of the posterobasal and laterobasal segments of the left lower lung lobe $(30 \times 15 \mathrm{~mm})$ as well as on the diaphragmatic surface $(37 \times 21 \mathrm{~mm})$ (Figures 1,2).

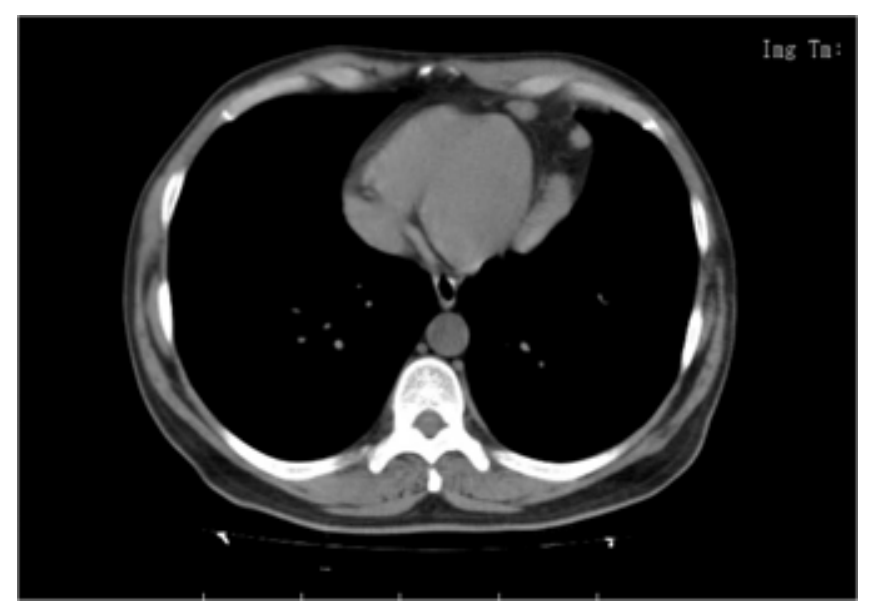

Figure 1. Thoracic computed tomography showing nodular lesions of soft tissue density in the pleural region.

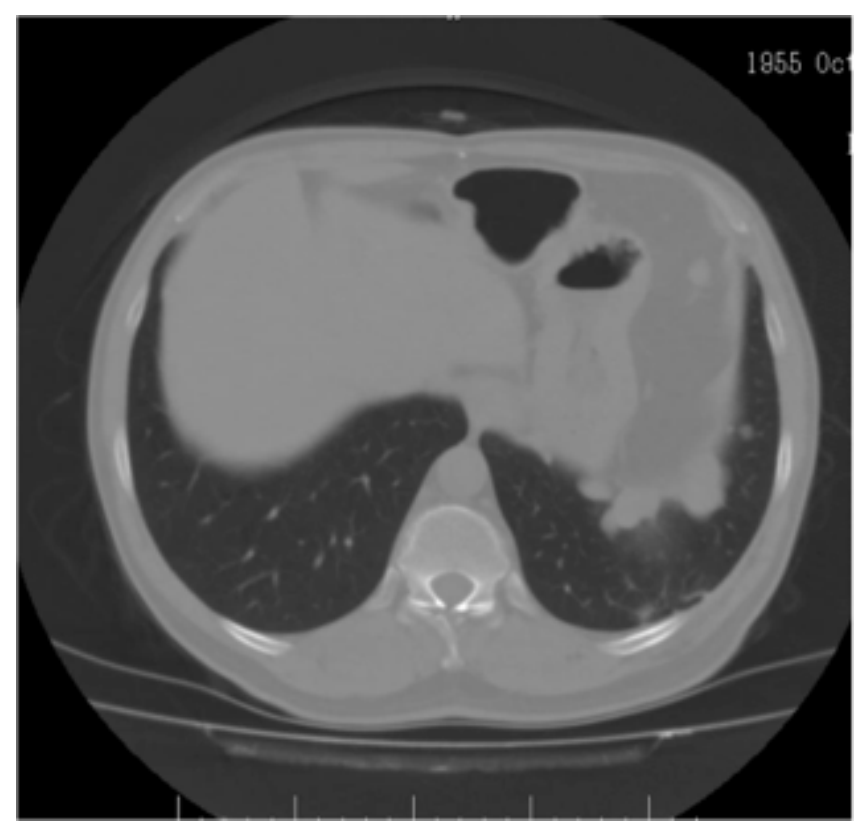

Figure 2. Thoracic computed tomography showing similar lesions on the diaphragmatic surface.
The patient's laboratory test results and respiratory function were normal. He had a history of splenectomy after a gunshot wound 25 years previously. Diagnostic video-assisted thoracoscopic surgery (VATS) revealed multiple dark red, hemorrhagic, fragile masses on the paracardiac, diaphragmatic and mediastinal surfaces. One of these masses on the paracardiac and diaphragmatic surfaces was dissected using a harmonic scalpel. Frozen section analysis revealed a possibly malignant lymphoid mass. No further procedures were performed because all lesions were suspected to be malignant. Postoperative pathological examination revealed ectopic splenic tissue. The activity on postoperative Tc-99m labeled heat-damaged erythrocyte scintigraphy showed accessory splenic tissue in the abdominal cavity, splenic area, perisplenic tissue, anterior midline, lower aspect of the liver, left lower lung lobe, and infrahilar region (Figure 3). The patient had no further symptoms and is continuing to be followed up.

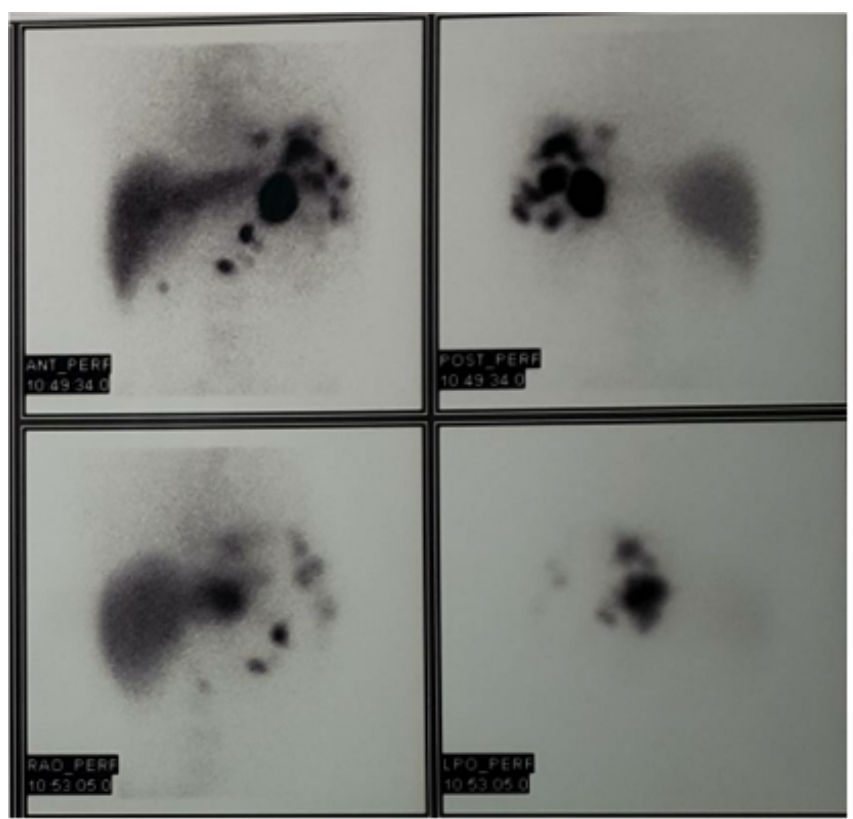

Figure 3. Tc-99m heat-damaged erythrocyte scintigraphy image showing accessory splenic tissue

\section{Discussion}

Thoracic splenosis may occur after blunt or penetrating trauma of the thoracoabdominal region. It is diagnosed in $65 \%$ of cases of splenic rupture [1]. Trauma-related splenosis is more frequent in the peritoneum, omentum, and mesenterium. The incidence of intrathoracic splenosis is $18 \%$, and the most common location is the pleural space of the left hemithorax [2]. 
Thoracic splenosis was first described by Shaw and Shafi in 1937. They defined thoracic splenosis as the autotransplantation of splenic tissue secondary to splenectomy or iatrogenic traumatic injury. The average time interval between the initial trauma and diagnosis is $>18.8$ years [3]. The patient's history of splenectomy due to an abdominal gunshot injury 25 years previously was the cause of the thoracic splenosis. Investigation of the patient's background is important to obtain appropriate differential diagnoses of multiple lesions in the thorax and abdomen.

Thoracic splenosis presents as sessile or pedunculated beefy reddish-blue lesions ranging from a few millimeters to $7 \mathrm{~cm}$ in diameter [4]. Pleuritic chest pain or the development of hemoptysis may support the diagnosis. Two factors are significant for a correct diagnosis: the presence of few asymptomatic left pleural-based nodules and a history of thoracoabdominal trauma requiring splenectomy. The lesions may also be approached by fine needle aspiration [5], but the presence of lymphocytic infiltration might lead to a misdiagnosis of lymphoma.

Abdominal trauma, especially a history of splenectomy, and multiple left pleural-based nodules should lead to a diagnosis of thoracic splenosis. The lesions found in the patient were diagnosed after chest pain had developed. A history of a malignancy in such patients might increase the complexity of achieving a correct diagnosis.

VATS is an option for patients with these types of multiple lesions. The causes of chest pain include the mass effect and irritation of the pericardium, parietal pleura, and diaphragm via the phrenic nerve [3]. The vessels in autotransplanted splenic tissue differ from the local perforator arteries arising from the splenic artery.

The use of Tc-99m sulfur colloid, indium-111-labeled platelets, or Tc-99m heat-damaged erythrocyte scintigraphy helps the splenic tissue to uptake radioactive isotopes [6]. Multiple specific regions of uptake were observed in the left hemithorax (Figure 3).
Pathological evaluation might rule out lesions such as metastasis (mostly that originating from lung carcinoma, breast carcinoma, or melanoma), lymphoma, a fibrous tumor of the pleura, invasive thymoma, and malignant mesothelioma [7].

As a conclusion thoracic splenosis should be considered in the differential diagnosis of patients with multiple lesions in the left hemithorax, and a history of splenic trauma involving thoracoabdominal injury.

\section{Declaration of conflicting interests}

The author declared no conflicts of interest with respect to the authorship and/or publication of this article.

\section{Funding}

The author received no financial support.

\section{References}

1. Malik UF, Martin MR, Patel R, Mahmoud A. Parenchymal thoracic splenosis: history and nuclear imaging without invasive procedures may provide diagnosis. J Clin Med Res 2010; 2: 180-74.

2. O-Yurvati AH, Thompson JB, Woods TN. Thoracic splenosis more than 40 years after thoracoabdominal trauma. J Am Osteopath Assoc 2013; 113: 853-6.

3. Fukuhara S, Tyagi S, Yun J, Karpeh M, Reyes A. Intrathoracic splenosis presenting as persistent chest pain. J Cardiothorac Surg 2012; 7: 84.

4. Moncada R, Wilams V, Fared J, Mesmore H. Thoracic splenosis. AJR Am J Roentgenol 1985; 144: 705-6.

5. Syed S, Zaharopoulos P. Thoracic splenosis diagnosed by fine needle aspiration cytology: a case report. Diagn Cytopathol 2001; 25: 321-4.

6. Huang AH, Shaffer K. Case 93: thoracic splenosis. Radiology 2006; 239: 293-6.

7. Kim K, Choi HJ, Kim YM, Kwon WJ, Lee WC, Suh JH. Thoracic splenosis: a case report and the importance of clinical history. J Korean Med Sci 2010; 25: 299-303. 